\author{
Pattern Recognition Letters \\ journal homepage: www.elsevier.com
}

\title{
Self-Adaptive SVDD integrated with AP clustering for one-class classification
}

\author{
Tong Wu $\mathrm{W}^{\mathrm{a}}$, Yanchun Liang ${ }^{\mathrm{a}, \mathrm{b}}$, RamiroVarela ${ }^{\mathrm{c}}$, Chunguo Wu ${ }^{\mathrm{a}}$, Guozhong Zhao ${ }^{\mathrm{d}}$ and Xiaosong Han ${ }^{\mathrm{a}, \mathrm{c}, \mathrm{d}, \mathrm{e}, *}$ \\ ${ }^{a}$ College of Computer Science and Technology, Key Laboratory of Symbolic Computation and Knowledge Engineering of Ministry of Education, Jilin University, \\ Changchun 130012, China \\ ${ }^{b}$ Department of Computer Science and Technology, Zhuhai Laboratory of Key Laboratory of Symbolic Computation and Knowledge Engineering of Ministry of \\ Education, Zhuhai College of Jilin University, Zhuhai 519041, China \\ ${ }^{c}$ Department of Computing, University of Oviedo, Campus of Gijón, 33204, Spain \\ ${ }^{d}$ Exploration and Development Research Institute, Daqing Oilfield Company, CNPC, Daqing 163712, China \\ ${ }^{e}$ Talent Development Institute, Daqing Oilfield Company, CNPC, Daqing 163000, China
}

\section{ABSTRACT}

The Support Vector Data Description (SVDD) is one of the best-known one-class-classification methods used to solve problems where the sample data are of high dimension but limited amount. The results of SVDD can be greatly affected when the target data are poorly distributed and their density varies extremely. To deal with situations like these, we propose an improved SVDD algorithm, termed as Self-Adaptive SVDD (SA_SVDD), which combines Affinity Propagation (AP) clustering algorithm and SVDD. Firstly, SA_SVDD applies AP clustering to the input data set to obtain a set of compact subclasses, and then the boundary for each subclass is identified by SVDD, and these boundaries are exploited as final classification criteria. AP clustering is tried with different input preferences generated with Latin Hypercube Sampling, and an improved Particle Swarm Optimization (PSO) algorithm evolves the parameters of SVDD. The proposed algorithm is evaluated on a conventional benchmark set and shows significant improvement with respect to other one-class-classification methods.

Keywords: one-class classifier; SVDD; AP clustering; parameters selection

2012 Elsevier Ltd. All rights reserved.

\section{Introduction}

One-class classification problem assumes that the target data are only used to describe a closed boundary, which distinguishes the target data and the outliers (Tax, 2001; Wu et al., 2011). To address one-class classification problem several methods have been proposed, which may be classified in density estimation (Juszczak P, 2006), clustering and support domain (Tax and Duin, 1999). Methods based on density estimation evaluate the density of the training data and find the threshold value to judge the class of the testing set, the most frequently-used estimation methods being Gaussian (Bishop, 1995) and Parzen window probability density estimation (Bishop, 1994) In accordance with the distribution of the samples, clustering-based methods distribute the samples into several subclasses firstly, and then calculate a threshold as the average distance of all sample data to its cluster center, which is used to classify new samples. The one-class classification methods based on the support domain usually describe a minimum boundary surface of target data. One-class Support Vector Machine (OCSVM) and Support Vector Data Description (SVDD) belong to this class and are widely used (Pan et al., 2009).

\footnotetext{
* Corresponding author. Tel.:+86 1331430 3309; e-mail: hanxiaosong@jlu.edu.cn
}

SVDD was developed by Tax in 1999 (Tax and Duin, 1999). The basic idea of the algorithm is to find a minimum spherically boundary covering all samples of the target class; this hypersphere is then used as the classifier to solve the one-class classification problem (Tax and Duin, 2004). Currently, one-class classification is applied in many research fields, such as network traffic anomaly detection, fault diagnosis, novelty detection and credit card fraud detection (Zhao et al., 2013; Hejazi and Singh, 2013; Hansen et al., 2010; Araki et al., 2014). With the deep study of one-class classification, many researchers put forward improved strategies in pursuit of higher efficiency and classification performance. Manevitz (Li, 2011; Manevitz and Yousef, 2001) claims that the boundary of the classifier can be decided by minority nonzero support vectors. Therefore, support vector samples may be selected to construct a reduced and so efficient training set. Besides the scale of the sample set, classification performance of training models also depends on their parameters. To adjust them, Zhang Zongsheng (Wu et al., 2013) proposed the weight value and dynamic inertia weight, which is based on original simulated annealing algorithm and particle swarm optimization. They demonstrated the high performance of their optimization strategy in the web traffic classification problem. At the same time, Chen Derong (Xu et al., 2010; Kan et al., 2008) proposed the idea of parallel learning: the training set is firstly divided into several subclasses by K-means algorithm, and then SVDD is applied to describe the closed boundary for each subclass. Thus, the problem of large scale 
dataset with great noise and lower density is solved, and the classification accuracy is also improved.

However, there are still some problems unsettled. One of them is that parameters selection is quite complex and usually depends on experience. Traditional clustering algorithms often need to specify the number of clusters in advance and so their results may strongly depend on the choice of the initial cluster centers (Jain, 2010). Moreover, the cluster centers and the number of clusters are dynamic in different datasets, which means that we should set appropriate initial parameters for various datasets. This process will bring heavy calculation and so it will reduce efficiency. To overcome these problems, we propose to fuse AP clustering (Frey and Dueck, 2007), global prediction-based adaptive mutation particle swarm optimization (GPAM-PSO) (Li et al., 2014) and SVDD into the so called Self-Adaptive Support Vector Data Description (SA_SVDD) algorithm.

The remaining of the paper is organized as follows. In Section 2, we introduce the two main methods used in SA_SVDD, namely SVDD, AP clustering and GPAM-PSO. In Section 3, we briefly review two classic methods for one-class classification and a novel clustering approach. In Section 4, we describe the proposed SA_SVDD algorithm and show how this algorithm adjusts the parameters self-adaptively. In Section 5, we report the results of the numerical experiments performed to evaluate our algorithm. At last, Section 6 gives the conclusions.

\section{Background}

In this section, we review the SVDD model, the AP clustering and the GPAM-PSO algorithm, as they are the basic components of the proposed SA_SVDD. AP is used to divide complex structural datasets into several simple sub-datasets, and then SVDD is employed to describe the boundary of each sub-dataset with optimization of GPAM-PSO.

\subsection{SVDD model}

Let us consider an $n$-dimensional training set $\left\{x_{i} \mid x_{i} \in R^{n}\right\}$. In the SVDD model (Tax and Duin, 1999; Tax and Duin, 2004), it tries to find the minimum hypersphere that contains as much of the target data as possible while rejects most of the outliers in the feature space. The hypersphere is defined by the center $a$ and radius $R$. Formally, the model of SVDD can be defined as the following optimization problem:

Minimize: $F(R, a)=R^{2}+C \sum_{i} \xi_{i}$

Subject to: $\left\|x_{i}-a\right\|^{2} \leq R^{2}+\xi_{i}, \xi_{i} \geq 0, \forall i$

where $\xi_{i}$ is the slack variable and represents the error estimation of $x_{i}$, and $C$ is a penalty factor that may be used to control the number of the bearable outliers. Eqs.(2) can be incorporated into eqs. (1) with the help of Lagrange multipliers as:

$L=R^{2}+C \sum_{i} \xi_{i}-\sum_{i} \alpha_{i}\left\{R^{2}+\xi_{i}-\left(\left\|x_{i}-a\right\|^{2}\right)\right\}-\sum_{i} \gamma_{i} \xi_{i}$

where $\alpha_{i} \geq 0, \gamma_{i} \geq 0$, in order to minimize the Lagrangian. Calculating partial derivatives with respect to $R, a$ and $\xi_{i}$ and letting them to be 0 , we obtain:

$\sum_{i} \alpha_{i}=1, a=\sum_{i} \alpha_{i} x_{i}, \quad 0 \leq \alpha_{i} \leq C$

The data are projected onto a higher dimensional feature space via the kernel function $\phi$, which transforms the linearly inseparable problem into a separable one. So, in accordance with eqs. (3) and (4), the dual optimization problem can be defined as:

$$
L=\sum_{i} \alpha_{i} \phi\left(x_{i}, x_{i}\right)-\sum_{i, j} \alpha_{i} \alpha_{j} \phi\left(x_{i}, x_{j}\right)
$$

Subject to: $\sum_{i} \alpha_{i}=1,0 \leq \alpha_{i} \leq C$ where $\phi\left(x_{i}, x_{i}\right)$ is the kernel function. The condition $0<\alpha_{i}<C$ means that the sample data are located on the boundary of the hypersphere, named support vector. For $\alpha_{i}=0$, the sample data are in the hypersphere, and for $\alpha_{i}=C$ the sample data are outside the hypersphere. Therefore the testing sample $z$ can be estimated by the following discrimination function:

$f(z)=\phi(z, z)-2 \sum_{i} \alpha_{i} \phi\left(z, x_{i}\right)+\sum_{i, j} \alpha_{i} \alpha_{j} \phi\left(x_{i}, x_{j}\right)$

Finally, the label of sample $\mathrm{z}$ will be decided by the sign of $g(z)$ defined as:

$g(z)=\operatorname{sgn}\left(R^{2}-f(z)\right)=\operatorname{sign}\left(R^{2}-\|z-a\|^{2}\right)$

Therefore, the accuracy of the classifier will depend on the selected kernel function and the parameter $C$.

\subsection{AP clustering}

Affinity Propagation (AP) clustering was proposed by Frey and Dueck in 2007, since then it has been widely applied to many fields. AP takes as input data real-valued similarities, $s(i, k)$, between pairs of data points $i$ and $k$, and then real-valued messages are exchanged between data points until a high-quality set of exemplars and corresponding clusters gradually emerges. There are two kinds of messages exchanged between data points, each one taking into account a different kind of competition. Messages can be combined at any stage to decide which points are exemplars and, for every other point, which exemplar it belongs to. The first kind of message is called "responsibility", denoted as $r(i, k)$, which is sent from data point $i$ to candidate exemplar point $k$ and reflects the accumulated evidence for how well-suited data point $k$ is to serve as the exemplar for data point $i$. The second kind of message is called "availability", denoted as $a(i, k)$, which is sent from candidate exemplar point $k$ to point $i$ and reflects the accumulated evidence for how appropriate it would be for point $i$ to choose point $k$ as its exemplar. To begin with, the availabilities are initialized to zero, i.e., $a(i, k)=0$. Then, the responsibilities are computed using the rule:

$r(i, k) \leftarrow s(i, k)-\max _{k^{\prime} \neq k}\left\{a\left(i, k^{\prime}\right)+s\left(i, k^{\prime}\right)\right\}$

With this rule all candidate exemplars compete for ownership of a data point. At the same time, the following availability update rule gathers evidence from data points as to whether each candidate exemplar would make a good exemplar:

$a(i, k) \leftarrow \min \left\{0, r(k, k)+\sum_{i^{\prime} \neq i, k} \max \left\{0, r\left(i^{\prime}, k\right)\right\}\right\}$

s no longer changed, or the algorithm runs beyond the maximum iterations. The damping factor $\lambda$ is used to avoid shocking in AP. The "responsibility" and "availability" values are set to $\lambda$ times their last iteration values plus $1-\lambda$ times their prescribed updated values.

In section 5, several experiments show that AP can lead to better performance compared to other clustering algorithms. Unlike $K$-means, AP does not require that the number of clusters be pre-specified. However, it takes as input a real value $s(k, k)$ for each point $k$ termed "preference", so that points with larger preferences are more likely to be chosen as exemplars, and so these input preferences have influence on the number of clusters. If all points are considered equally suitable as exemplars, all input preferences should be set to a shared preference value $P$, which can be varied to produce different numbers of clusters.

\subsection{Analysis of preferences}

As pointed in Section 2.2, different $P$ values will lead to various clustering results. So, we will apply AP considering different $P$ values and use Silhouette (SIL) (Dudoit and Fridlyand, 
2002; Rousseeuw, 1987) index as validity evaluation function to identify the optimal classifier. Silhouette index is one of the internal validity indexes of common use, which describes the intra class compactness and the inter-class separability of the clustering structure. It is suitable for class-unknown data sets and for a point $i$ is defined as:

$s(i)=(b(i)-a(i)) /(\max \{a(i), b(i)\})$

where $a(i)$ denotes the average dissimilarity between the point $i$ and other points in its class, and $b(i)$ introduces the lowest average dissimilarity between point $i$ and all other classes. It is clear that $-1 \leq s(i) \leq 1$. For $s(i)$ to be close to 1 , we require $a(i) \ll b(i)$. The average $s(i)$ over all the sample data can be used to evaluate the clustering quality; its maximum value corresponding to the optimal number of clusters.

Regarding the $P$ value, it is usually set as the median of the elements in the main diagonal of the similarity matrix, but this value is not always the best one. Here, we use Latin Hypercube Sampling (LHS) (McKay et al., 1979) to find the optimal $P$ value.

LHS is a statistical sampling method for generating a random sample. When it works, firstly the problem will be divided into $M$ areas without overlapping, which each area has the same probability, finally select a point randomly in each area to form the final samples.

\subsection{GPAM-PSO}

GPAM-PSO was proposed by Li et al. (2014), and is able to improve the convergence speed and to avoid premature convergence at the same time, thanks to the characteristics of its global optimal predicting and self-adaptive mutation operations. An aggregation operator based on regression model allows GPAM-PSO to predict the optimal particle location of the current population, and a divergence operator is used to identify and mutate the particles that fell into local optima.

The flow of the algorithm is as the following:

1. Map the swarm to a lower feature space with the PCA;

2. In the lower feature space, construct the linear regression model for the above swarm data to predict the global optimal position. The regression function is:

$y_{i}=\sum_{j=0}^{m} \omega_{j} \varphi_{j}\left(z_{i}\right)+\varepsilon$

where $y_{i}$ is the objective function value of particle $x_{i}, \omega_{j}$ is the regression parameter, and the $\varphi_{j}\left(z_{i}\right)$ is the basis function, which is the quadratic polynomial function in this algorithm;

3. The global optimal position is mapped from the lower feature space onto the original space to guide the swarm evolution;

4. The aggregation operator and the divergence operator are imported to monitor the aggregation of particles, find the localtrapped particles, and help them escape from the local optimums.

Fig. 1. Procedure of GPAM-PSO

\section{Literature Review}

In this section, we review some methods related to our proposal with the purpose of establishing some similarities and differences and also to justify some of our choices. Schölkopf et al. (2001) proposed One Class Support Vector Machine (OCSVM). The basic idea is to construct a hyper-plane in the feature space, which tries to separate the target samples from the origin as far as possible. When the Gaussian kernel is used, both the SVDD and the OCSVM give comparable solutions (Tax and Duin, 2004). The parameters selection influences the performance of SVM classifiers greatly. For this reason, some researchers had tried to improve the performance of their classifiers by optimizing the method of parameters selection (Thomas et al., 2015; Xiao et al., 2013; Lian, 2012).

KM_SVDD (K-Means SVDD) was proposed by Tax in (Tax, 2001), and since then it was subsequently improved (Wang and Lai, 2013; Xu et al., 2011; Wang and Tan, 2013; Xiao, 2013; Hua et al., 2009; Wu, 2013). The basic idea is to divide the training set into some sub-datasets by $K$-means clustering, and then to describe the boundary of classifier through SVDD. The aim of KM_SVDD is to save the training time of SVDD by shrinking the size of the training set. However, since the number of clusters and their initial centers with $K$-means clustering are not determinate, the efficiency of the algorithm cannot be guaranteed.

The clustering approach developed by Rodriguez and Laio (2014), termed density peaks (DP) clustering in this paper, is a good algorithm to describe the data distribution by density peaks, and has lower complexity than traditional $K$-means clustering. In DP, the cluster centers are recognized as local density maxima that are far away from any points of higher density. DP depends only on the relative densities rather than on their absolute values. So, there is no need to map the points into the feature space. But the memory overhead of the distance matrix is too huge if the sample set is very large. Furthermore, the clustering centers need to be chosen according to the decision diagram manually, which obviously results in lack of automation.

\section{SA_SVDD}

\subsection{Algorithm description}

The classifier of a one-class classification problem is obtained by training a dataset which only contains one class. When the dataset distributes with variety densities, if their boundary is described with only one hypersphere as done in the SVDD, this boundary may not describe accurately, and will lead to a poor classification performance (Bao, 2011). This fact motivates the improved SVDD algorithm proposed in this paper. In this algorithm, the input data are firstly distributed into a number of $K$ clusters by AP. Then, it obtains $K$ hyperspheres by means of SVDD, and the parameters of SVDD are evolved by GPAM-PSO. In the end, all $K$ hyperspheres are used as the classification criterion to predict the membership of new sample data to the class. The training set is the only input of SA_SVDD, and all the relevant parameters, for example $P, K, C$ and all the parameters of the kernel function, which may influence the boundary of the hyperspheres, will be computed self-adaptively from the training set.

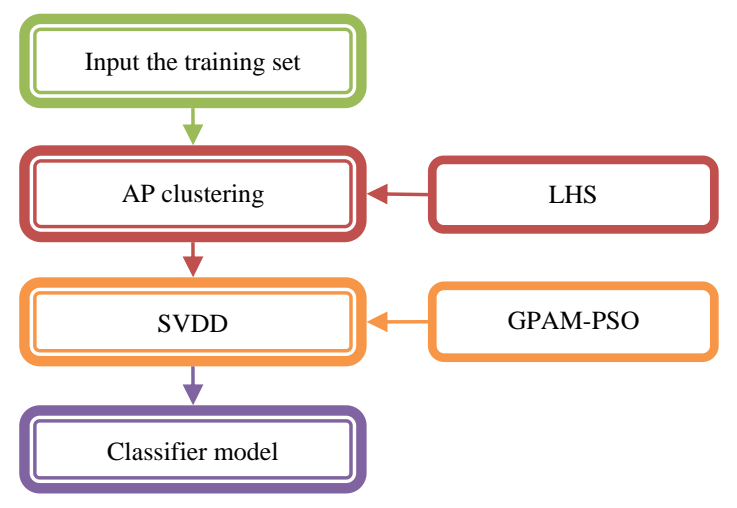

Fig. 2. Flowchart of SA_SVDD

SA_SVDD applies the divide-and-conquer approach to transform the problem of solving a large sphere into the problem of solving several smaller spheres, which can improve the 
precision and the efficiency of the classifier. The process of the SA_SVDD is shown in Fig 2, and its steps are detailed in Fig 3. Although the most important parameters will be calculated automatically, we still need to set some less important ones. For example, in AP algorithm, the damping factor $\lambda$ and the maximum number of iterations, in GPAM-PSO, the number of iterations, swarm size, the range of $C$ and $\sigma$, and the intervals of LHS are all need to be assigned. After setting these parameters, SA_SVDD will apply AP from the input dataset considering different $P$ values. To select candidate $P$ values, LHS and SIL index are used due to their simplicity and low time consumption. For each value, AP clustering is applied to obtain $K$ compacted sub-clusters, using Euclidean distance as the similarity metric. After that, SA_SVDD applies SVDD to each sub-cluster in the best set of clusters obtained in previous step. An improved PSO, GPAM-PSO, is employed in this step to evolve the best parameters of SVDD, because of its self-adaptability and ability of avoiding premature convergence. If the sample data are well distributed by AP, hopefully the boundaries established by the $K$ sub-hyperspheres will be much more accurate than the boundary given by only one hypersphere obtained from the whole sample data. The result of this step is a set of $K$ sub-hyperspheres, which will be used in the classification process: a new sample belongs to the class if it belongs to any of the $K$ sub-hyperspheres.

Fig.4 illustrates the main steps of the training process of SA_SVDD. The training data set contains 200 2-dimensional Banana data generated at random (Fig. 4a). AP clustering distributes these samples into 3 sub-clusters (Fig. 4b). Finally,

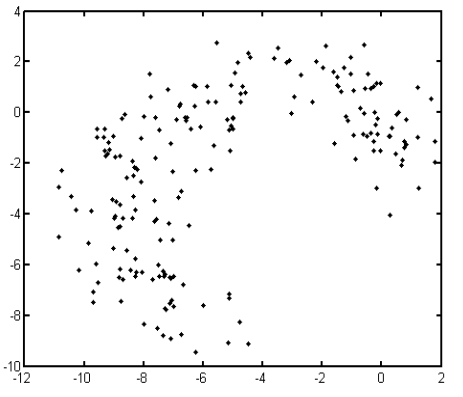

a) An artificial data set

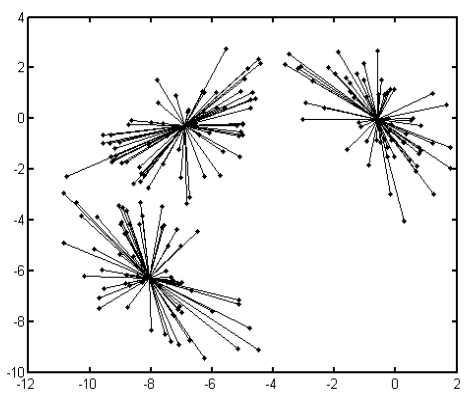

b) Result of AP clustering
Fig. 4c shows the boundaries of the sub-clusters obtained by SVDD from the best parameters obtained with GPAM-PSO.

\begin{tabular}{|l}
\hline Step 1. Initialization \\
- Input the training set; \\
Step 2. AP clustering \\
- Apply LHS to obtain a subset of candidate $P$ values \\
from the similarity matrix; \\
- Apply AP from all candidate $P$ values; \\
- Evaluate the clustering results with SIL index; \\
- Return the set of $K$ sub-clusters with largest SIL \\
index; \\
Step 3. Construction of classification hyperspheres; \\
- Apply GPAM-PSO to evolve the parameters of \\
SVDD for each sub-cluster, taking fitness as the \\
classification results of SVDD with 5-fold cross- \\
validation; \\
Return the $K$ sub-hyperspheres obtained from the \\
best parameters of SVDD for each one of the $K$ \\
sub-clusters.
\end{tabular}

Fig. 3. Procedure of SA_SVDD

In next sub-sections, we will discuss some of these optimization steps in more detail.

Fig. 4. The visualization process of SA_SVDD algorithm

\subsection{Parameter optimization}

In the algorithm, we need to set some important parameters, which greatly affect the performance, such as the preference of $\mathrm{AP}$, the penalty factor $C$ and the kernel function parameter $\sigma$. In order to achieve the self-adaptation of SA_SVDD, we design a few methods to select the parameters adaptively.

\section{1). Optimization of preferences}

We use LHS algorithm and the SIL index to find the optimal $P$ value. The main steps are as follows:

1. We consider the median in the first trial, and then use LHS to generate $\mathrm{m} / 2$ candidate $P$ values before and after the median respectively. Fig 6 shows the LHS process for $m=6$;

2. AP clustering is run for each $P$ value, and then SIL indexes are calculated to evaluate each clustering result, which obtains the collection $\left(\mathrm{S}_{1}, S_{2}, \ldots, S_{m}\right)$;

3. The candidate $P$ value giving rise to the largest SIL is taken as the best $P$ value and the results of AP with this $P$ value are the optimal set of clusters.

Fig.5. Process of preferences optimization

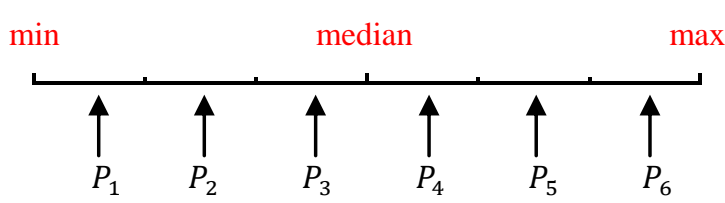

Fig. 6. Procedure of LHS, $m=6$

In the worst case, AP will iterate $\mathrm{m}$ times at most. However, if the SIL index is very close to 1 , the iterative process will terminate before $\mathrm{m}$ steps. For example, Table 1 shows the optimal numbers of clusters and the ratio between the best $P$ value and the median obtained for 6 datasets from the UCI repository and LIBSVM datasets(Chang and Lin,2001) taking $\mathrm{m}=40$ and finishing the iterative process as soon as the SIL index reaches 0.9 .

Table 1

The optimal number of clusters obtained by Silhouette

\begin{tabular}{lllll}
\hline Data sets & Instances & \#class & SIL & $P$ value / median \\
\hline breast_cancer & 683 & 2 & $\underline{2}$ & 38.95 \\
diabetes & 768 & 2 & $\underline{2}$ & 48.58 \\
dna & 2000 & 3 & $\underline{3}$ & 9.84 \\
iris & 150 & 3 & $\underline{3}$ & 4.1 \\
liver-disorders & 345 & 2 & $\underline{2}$ & 30.69 \\
vehicle & 946 & 4 & $\underline{4}$ & 14.53 \\
\hline
\end{tabular}


2).Optimization of $C$ and $\sigma$.

In the SVDD model, the penalty factor $C$ and the kernel function decide the boundary of the hypersphere. $C$ indicates how many outliers may be allocated in the hypersphere. If $C$ increases, the hypersphere enlarges. Following Tax (2001), we use the following Gaussian kernel function:

$k\left(x_{1}, x_{2}\right)=\exp \left(-\frac{\left\|x_{1}-x_{2}\right\|^{2}}{2 \sigma^{2}}\right)$

where $\sigma$ is the kernel-width parameter, which affects the number of support vectors and so it is crucial for the accuracy of the classifier.

Therefore, the final boundary of the classifier will depend on the parameters $C$ and $\sigma$. In SA_SVDD, as we obtain a number of sub-clusters, for the precise boundary of every sub-cluster, we will need optimal parameters to apply SVDD to each sub-cluster instead of the same parameter for all of them. To obtain these parameters, in this paper we use GPAM-PSO. We illustrate the improvement of GPAM-PSO with respect to other conventional methods, PSO and grid search, with the following preliminary experiment.

1. Select the Banana dataset of the Figure 4;

2. Use the grid search method, the PSO and the GPAM-PSO to optimize the parameters $C$ and $\sigma$ for the target data set (the result of 5-fold cross-validation of SVDD is set as the fitness function) and giving all these algorithms similar running time;

3. According to the best parameters obtained with each method, describe the 3 classification boundaries of the target dataset.

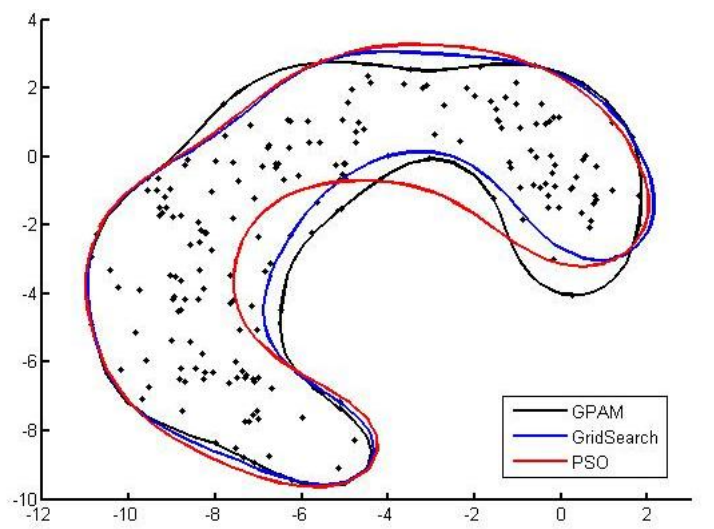

Fig. 7. Comparison of 3 kinds of optimization algorithm

In Fig 7, the black line, blue line and red line represent the boundaries after conducting GPAM-PSO, grid search and PSO respectively. It can be seen that the GPAM-PSO describes more compact and accurate boundary than the other two methods. This single experiment also demonstrates that the selection of parameters has a great influence on the performance of the classifier.

\section{Experimental results and analysis}

To evaluate SA_SVDD and for comparison with the state-ofthe-art methods mentioned in Section 3, we have conducted an experimental study on conventional data sets taken from the UCI repository (http://archive.ics.uci.edu/ml/) and the LIBSVM data
collection(Chang and Lin,2001). The SVDD and oneclass SVM in the experiments are both derived from the libsvm toolbox.

The program is implemented in Matlab R2013 and runs on PC (4G RAM and Core i5 2400), and it is hosted in GitHub (https://github.com/SASVDDwt/sa_svdd).

\subsection{Evaluation measurement}

As performance metrics we consider $F$-measure and boxplots. $F$-measure is commonly used to evaluate the accuracy of classifiers when the training data are imbalanced or poorly distributed. It is defined from the harmonic mean of precision $(P)$ and recall $(R)$ as:

$F=2 R P /(R+P)$

where $P$ and $R$ are calculated from the true positives $(T P)$, true negatives $(T N)$, false positives $(F P)$ and false negatives $(F N)$ obtained in the classification process as:

$P=T P /(T P+F P)$

$R=T P /(T P+F N)$

Therefore, $P$ denotes the proportion of the sample for which its real class and the predicted class are both positive, and recall $R$ represents the proportion of the correctly predicted sample. High recall means the classifier barely determines a positive class as negative one. Similarly, the classifier is less likely to determine a negative sample as positive when the precision is high.

\subsection{Comparison of SA_SVDD with other methods}

To verify the performance, we calculated the $F$-measures of SVDD、SA_SVDD and OCSVM on the 6 datasets.

For each dataset, the class containing the majority samples was selected as the positive class and the remaining classes were taken as the negative one. The positive class was randomly divided into two parts, one was used for training $(80 \%)$ and the rest part (20\%) combined with all the negative class for validating. Table 2 lists the detail information of datasets and the average density of training sets before and after the AP clustering, which is used to measure the distribution of datasets. The density $\rho_{i}$ of data point $i$ is defined as:

$\rho_{i}=\sum_{j} \chi\left(d_{i j}-d_{c}\right) / N$

where $\chi(x)=1$ if $x<0$ and $\chi(x)=0$ otherwise, $d_{i j}$ is the distances of between data points, and $d_{c}$ is a cutoff distance, $N$ is the sample size for normalization. We can observe that the subclass is used to describe a more compactness description than the initial dataset in the Table 2 .

We also substituted $K$-means and DP clustering for AP in SA_SVDD to test the effect of different clustering methods. KM_SVDD and DP_SVDD methods are same as SA_SVDD except the clustering method. In KM_SVDD, the initial clustering number of was set as the result of AP, therefore it is in advantage w.r.t. both DP_SVDD and SA_SVDD. GPAM-PSO algorithm was used in all five methods for parameter optimization. Table 2 lists the parameter setting method. Table 4 lists the average value from 30 times 5-fold cross-validation $F$ measure. 
Table 2.

Dataset discription

\begin{tabular}{llcccccc}
\hline \multirow{2}{*}{ Origin } & Dataset & \multirow{2}{*}{ Features } & \#Total(pos/neg) & \#Train(pos) & \#Validate(pos/neg) & density before AP & density after AP \\
\hline \multirow{3}{*}{ UCI } & breast_cancer & 10 & $683(444 / 239)$ & 355 & $328(89 / 239)$ & 0.0358 & 0.0433 \\
\cline { 2 - 9 } & ionosphere & 34 & $351(225 / 126)$ & 180 & $171(45 / 126)$ & 0.0673 & 0.0680 \\
\cline { 2 - 9 } & pima_indians_diabetes & 8 & $768(500 / 268)$ & 400 & $368(100 / 268)$ & 0.0716 & 0.0764 \\
\cline { 2 - 9 } & spambase & 57 & $4601(2788 / 1813)$ & 2230 & $2371(558 / 1813)$ & 0.0482 & 0.0623 \\
\hline \multirow{2}{*}{ LIBSVM } & svmguide3 & 21 & $1284(947 / 337)$ & 758 & $526(189 / 337)$ & 0.0811 & 0.0909 \\
\cline { 2 - 9 } & w1a & 300 & $2475(2405 / 72)$ & 1924 & $553(481 / 72)$ & 0.0822 & 0.0938 \\
\hline
\end{tabular}

Table 3.

Parameters setting

\begin{tabular}{lll}
\hline Algorithm & Parameters & Value \\
\hline \multirow{3}{*}{ GPAM- } & \#SO iteration & 200 \\
\cline { 2 - 3 } & \# particle & 20 \\
\cline { 2 - 3 } & range of $C$ & $10^{-4} \sim 1$ \\
\cline { 2 - 3 } & range of $\sigma$ & $10^{-4} \sim 10^{2}$ \\
\hline \multirow{3}{*}{ AP } & damping factor $\lambda$ & 0.9 \\
\cline { 2 - 3 } & \# iteration & 100 \\
\cline { 2 - 3 } & preference & from optimization \\
\hline LHS & \#Interval & 40 \\
\hline K-means & \#cluster & the same as AP \\
\hline
\end{tabular}

Table 4.

Comparison with different classification algorithms

\begin{tabular}{lrllll}
\hline \multirow{2}{*}{\multicolumn{1}{c}{ Dataset }} & \multicolumn{5}{c}{$\boldsymbol{F}$-measure (\%) } \\
\cline { 2 - 6 } & SVDD & One-class & KM_SVDD & DP_SVDD & SA_SVDD \\
\hline breast_cancer & $81.60 \%$ & $85.53 \%$ & $88.31 \%$ & $82.82 \%$ & $91.52 \%$ \\
\hline ionosphere & $58.32 \%$ & $63.84 \%$ & $75.74 \%$ & $66.49 \%$ & $76.56 \%$ \\
\hline pima_indians_diabetes & $43.13 \%$ & $43.52 \%$ & $43.50 \%$ & $43.46 \%$ & $44.48 \%$ \\
\hline spambase & $65.85 \%$ & $65.55 \%$ & $66.48 \%$ & $65.91 \%$ & $66.65 \%$ \\
\hline svmguide3 & $56.27 \%$ & $56.18 \%$ & $56.29 \%$ & $56.38 \%$ & $56.77 \%$ \\
\hline w1a & $85.63 \%$ & $88.83 \%$ & $82.30 \%$ & $88.42 \%$ & $91.07 \%$ \\
\hline
\end{tabular}

Use the $F$-measure as the validation index, the results of the 5 different classification algorithms are displayed with the boxplot in the Fig 8. As we can see in Table 4 and Fig 8 which shows the average performance of boxplot with five classification methods on the 6 considered datasets, it is clear that SA_SVDD performs better than the other methods. We can also observe that KM_SVDD shows $F$-measure values much better than DP_SVDD and very close to SA_SVDD. However, we have to take into account that this is due to giving KM_SVDD an accurate initial number of clusters. We have also experimented with KM_SVDD starting from random clusters and the results were clearly worse. This is due to the poor stability of $K$-means clustering. On the contrary, the results of AP clustering does not change no matter how many times AP is run on the same dataset.

However, we have to be aware that KM_SVDD, DP_SVDD and SA_SVDD are more time-consuming than SVDD alone and OCSVM. This is because SVDD has to be run many times for convergence. Yet, SVDD running in some small-scale datasets rather than a whole dataset avoids training massive dataset, and it also saves some time.

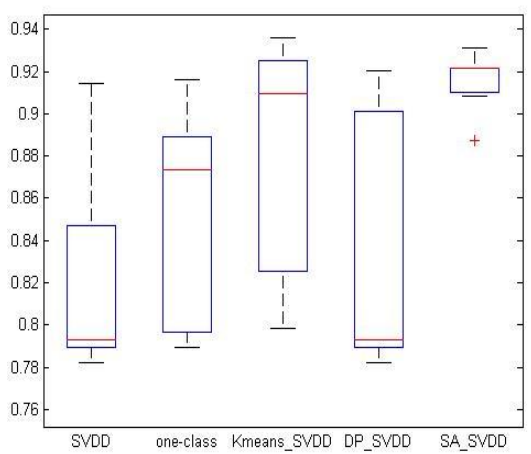

a) breast-cancer

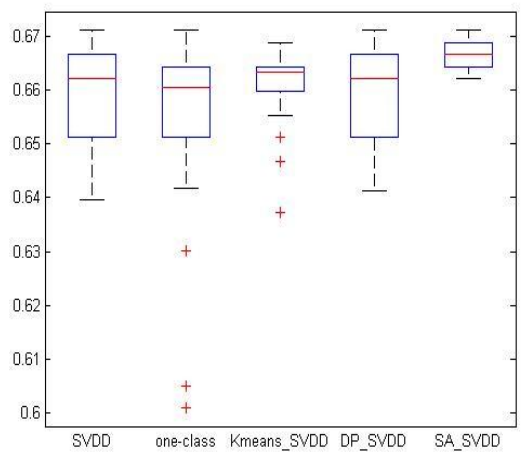

d) spambase

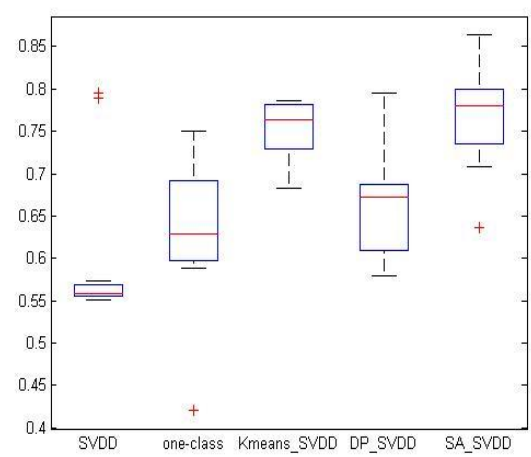

b) ionosphere

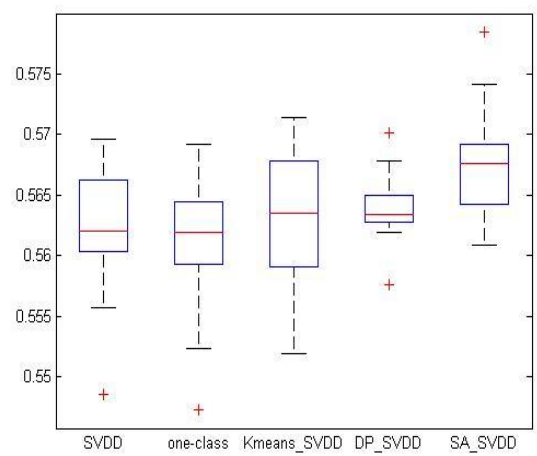

e) svmguide3

Fig. 8. Boxplot with different classification methods

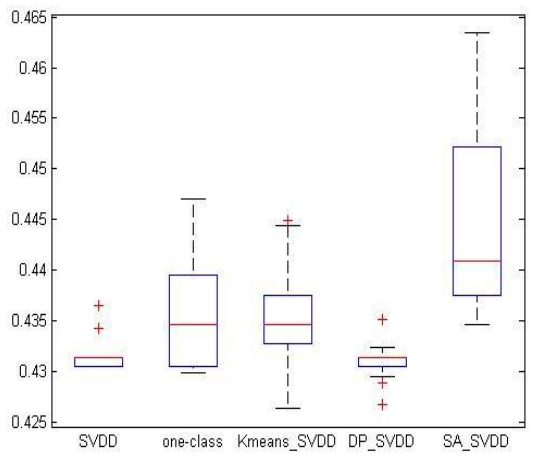

c) pima_indians_diabetes

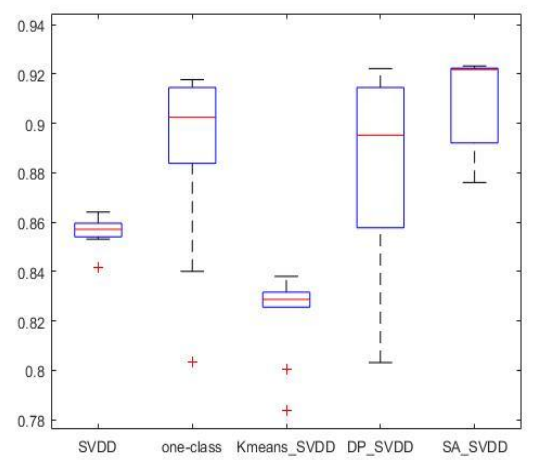

f) $w 1 a$ 


\section{Conclusions}

We have proposed an improved one-class classification method, termed SA_SVDD, which is able to deal with some common issues in traditional clustering such as poorly distributed data or loose boundary. The advantages of the proposed method over other conventional methods are derived from a proper combination of AP clustering and SVDD boundary detection, which is based on the idea of divide and ruling: the training set is divided into several subsets by AP, and then the boundary of each subset is determined by SA_SVDD. Experiments using artificial data and benchmark datasets show clearly that, compared with other classification methods in the state of the art, SA_SVDD improves the classification performance significantly.

Even though SA_SVDD takes larger time than other simple methods, as OCSVM or SVDD running alone, this time is still low enough considering it can be applied to solve real-life problems such as machine fault detection, network intrusion detection, and image analysis.

As future work we plan to keep studying proposed SVDDs, and further understand their advantages. Especially, we will try to find a proper trade-off between high $F N$ and high $N N$. Moreover, in accordance with the size of each subset, different optimistic methods will also be considered.

\section{Acknowledgments}

This work was supported by the National Science Foundation of China under Grant 61503150, 61472158, 61572228.

\section{References}

Araki, S., Yamaguchi, Y., Shimada, H., Takakura, H. 2014 Unknown Attack Detection by Multistage One-Class SVM Focusing on Communication Interval. 21st International Conference on Neural Information Processing (ICONIP), 325-332.

Bao, L., Huang, S.G., Li, Y.C., 2011. Multi-class classification method based on K-means cluster and hyper-sphere. Application Research of Computers, 28(5), 1764-1766.

Bishop, C., 1994. Novelty detection and neural network validation. IEEE Proceedings Vision, Image and Signal Processing, London: IEEE press, 217-222.

Bishop, C., 1995. Neural Networks for Pattern Recognition. New York: Oxford University Press.

Chang, C.C., Lin, C.J., 2001. LIBSVM:A library for support vector machines. Software available from:http://www.csie.ntu.edu.tw/ cjlin/libsvm.

Dudoit S, Fridlyand J., 2002. A prediction-based resampling method for estimating the number of clusters in a dataset .Genome Biology, 3(7), 121.

Frey, B. J., Dueck, D., 2007. Clustering by passing messages between data points. Science, 315(5814), 972-976.

Hansen, M. S., Sjöstrand, K., Larsen, R., 2010. On the regularization path of the support vector domain description. Pattern Recognition Letters, 31(13), 1919-1923

Hua, 2009. Updated Learning Algorithm of Support Vector Data Description Based on K-Means Clustering. Computer Engineering.35(17), 184-186.

Jain, Anil. K., 2010. Data clustering: 50 years beyond K-means. Pattern Recognition Letters, 31(8), 651-666.
Juszczak, P., 2006. Learning to recognize: A study on one-class classification and active learning. Delft University of Technology, Holland.

Kan, D.R., Gong, J.L., Chen, Q., Cao, X.P. 2008. Support vector data description for fast anomaly detection in hyperspectral imagery based on sample segmentation. Acta Armamentaria, 29(9), 1049-1053.

Lian, H., 2012. On feature selection with principal component analysis for one-class SVM. Pattern Recognition Letters, 33(9), 1027-1031.

Li, Q.Y., Li, G.Y., et al. Global Prediction-Based Adaptive Mutation Particle Swarm Optimization. 10th International Conference on Natural Computation, pp. 269-274.

Li, Y.H.,2011. Selecting training points for one-class support vector machines [J]. Pattern Recognition Letters, 2011, 32(11), 1517-1522.

Manevitz, L. M., Yousef, M., 2001. One-class SVMs for document classification. Journal of Machine Learning Research, 2(2001), 139-154.

McKay, M.D., Beckman, R.J., Conover, W.J., 1979. A Comparison of Three Methods for Selecting Values of Input Variables in the Analysis of Output from a Computer Code. Technometrics (JSTOR Abstract) (American Statistical Association), 21(2), 239-245.

Pan, Z.S., Chen, B., Miao, Z.M., Ni, G.Q., 2009. Overview of one-class classifiers. Chinese Journal of Electronics, 37(11), 2496-2503.

Rodriguez, Alex., Laio, Alessandro., 2014. Clustering by fast search and find of density peaks. Science, 344(6191), 1492-1496.

Rousseeuw, PJ., 1987. Silhouettes: a Graphical Aid to the Interpretation and Validation of Cluster Analysis. Computational and Applied Mathematics, 20, 53-65.

Schölkopf, B., Platt, J. C., Shawe-Taylor, J., et al., 2001. Estimating the support of a high-dimensional distribution. Neural Computation, 13(7), 1443-1471.

Singh, YP., 2013. One-class Support Vector Machines Approach to Anomaly Detection. Applied Artificial Intelligence, 27(5), 351-366.

Tax, D. M. J., Duin, R. P. W., 1999. Support vector domain description. Pattern Recognition Letters, 20(11-13), 1191-1199.

Tax, D., 2001 One-class classification: Concept-learning in the absence of counter-examples. Delft University of Technology, Holland.

Tax, D. M. J., Duin, R. P. W., 2004. Support Vector Data Description. Machine Learning Research, 54(1), 45-56.

Thomas, A., Feuillard, V., Gramfort, A., 2015. Calibration of One-Class SVM for MV set estimation. arXiv preprint arXiv:1508.07535.

Wang, C. D., Lai, J. H., 2013. Position regularized Support Vector Domain Description. Pattern Recognition,46(3),875-884.

Wang, D., Tan, X. Y., 2013. Centering SVDD for Unsupervised Feature Representation in Object Classification. Neural Information Processing. Springer Berlin Heidelberg, 2013, 376-383.

Wu, D.H., Zhang, P.L., Ren, G.Q., Chen F., 2011. Review of one-class classification method on support vector. Computer Engineering, 37(5), 187-189.

Wu, Q., Liu, J.N., Kou, W.L., Zhang, Z.S, 2013. Internet traffic identification by using improved one class support vector machines. Journal of Jilin University Engineering and Technology Edition, 43, 124-127.

Wu, M., 2013. Study on On-line Monitoring System and Method of Fault Diagnosis Based on Improved SVDD. Beijing University of Posts and Telecommunications.

Xiao, T., 2013. An Improved Support Vector Data Description Algorithm. Harbin Engineering University.

Xiao, Y., Wang, H., Zhang, L., et al.,2014. Two methods of selecting Gaussian kernel parameters for one-class SVM and their application to fault detection Knowledge-Based Systems, 59(2), 75-84.

Xu, J., Yao, J., Ni, L., 2011. Fault detection based on SVDD and cluster algorithm. Electronics, Communications and Control (ICECC), 2011 International Conference on. IEEE, 2011, 2050-2052.

Xu, J., Shi, R.Y., Zhan, Y.Z., Jiang, P., 2010. Model of IDS based on SVDD and cluster algorithm. Control and Decision, 25(3), 441-444.

Zhao, Y., Wang, S.W., Xiao, F., 2013. Pattern recognition-based chillers fault detection method using Support Vector Data Description (SVDD). 4th International Conference on Applied Energy, 112, 1041-1048.Hejazi, M., 